\title{
Consistency in the reporting of sexual behaviour by adolescent girls in Kenya: a comparison of interviewing methods
}

\author{
P C Hewett, B S Mensch, A S Erulkar
}

Sex Transm Infect 2004;80(Suppl II):ii43-ii48. doi: 10.1136/sti.2004.013250

See end of article for authors' affiliations ......................

Correspondence to: Dr P C Hewett, Policy Research Division Population Council, One Dag Hammarskjold Plaza, New York, NY 10017; phewett@popcouncil.org

Accepted for publication 16 September 2004

\begin{abstract}
Objectives: To investigate in a district in Kenya the level and consistency of reporting of sexual behaviour among adolescent girls randomly assigned to two modes of survey interview: face to face interview and audio computer assisted self-interview (ACASI).

Methods: The analysis is based on a subsample of over 700 never married girls aged 15-21 years in Kisumu, Kenya, drawn from a population based survey of over 2100 respondents. A questionnaire with 69 questions was used, two thirds of which were considered sensitive, including questions about risky sexual behaviour, alcohol and drug use, contraceptive practice, pregnancy, induced abortions, and births. Results: ACASI produced significantly higher reporting of sex with a relative, stranger, or older man, and higher reporting of coerced sex. However, differences by mode for ever had sex and sex with a boyfriend were not significant. Relative to ACASI, the interviewer administered mode produced highly consistent reporting of sexual activity, both within the main interview and between the main and exit interviews.

Conclusions: Both the mode of survey administration and the probing for various behaviours significantly affect the observed prevalence of sexual activity. The ACASI results suggest that adolescent girls in Kenya have more complex and perilous sex lives than traditional face to face surveys of sexual activity indicate. The level of consistency in the interviewer mode is argued to be suspect, particularly given the much lower levels of reporting, relative to ACASI, for types of sexual partners and coerced sexual activity.
\end{abstract}

n sub-Saharan Africa, where the major route of transmission of HIV is through heterosexual intercourse and where levels of infection among young people, particularly adolescent girls, are high, the accuracy of information on sexual activity prior to marriage is critically important for policy and programme purposes. ${ }^{12}$ Yet recent studies from the region raise questions about the validity of reporting of sexual behaviour. For instance, an analysis of trends in age at first sex in Africa using the Demographic and Health Surveys revealed discrepancies in reporting within birth cohorts, consistent with young women denying and young men exaggerating their sexual activity. ${ }^{3}$ An epidemiological investigation of the large gender disparity in HIV prevalence among young people in two African cities revealed HIV prevalence to be high among women reporting one sexual partner and few episodes of sexual intercourse, a pattern suggesting considerable underreporting of risky sexual behaviour among young women. ${ }^{4}$

These concerns about data quality underscore the importance of identifying the optimal mode of collecting information from young people. In previously published research on the effects of interview mode on the reporting of sensitive behaviour in Nyeri and Kisumu districts in Kenya, we evaluated whether audio computer assisted self-interviewing (ACASI) produced more credible data than interviewer administered and paper and pencil self-administered interview methods, using survey data from more than 6000 never married young people aged 15-21 years. ${ }^{5}$ The results from the first district, Nyeri, were, for the most part, inconsistent with our expectations. For instance, we anticipated that adolescent girls assigned to ACASI would report more premarital sex because that interviewing method was presumed to provide greater privacy in which to answer sensitive questions. Yet respondents were significantly more likely to report having had sex in face to face interviews. The findings for boys, although more in line with expectations, were not strong or consistent enough to suggest that ACASI was the preferred method of interviewing. The results from Kisumu were more in accordance with expectations. For the most part, ACASI generated significantly higher levels of reporting of a range of sensitive behaviours among girls-particularly among girls enrolled in school. For boys, who we believe exaggerate their level of sexual activity in face to face interviews, the reporting of girlfriends and premarital sex was lower with ACASI whereas reporting of more stigmatised behaviours was higher.

In this paper we explore the consistency of reporting of sexual behaviour among never married female respondents in Kisumu. We take advantage of the fact that in Kisumu, in contrast to Nyeri, detailed sexual behaviour questions were asked of every respondent, even those who answered negatively to an initial question about ever having had sexual intercourse. We also compare responses to questions about sexual behaviour in the main interview with responses in an exit interview where all respondents, including those in the ACASI group, were given a face to face interview.

\section{BACKGROUND AND LITERATURE}

Computer assisted interviewing technologies have been in existence for over three decades. ${ }^{67}$ With the introduction of personal computers, computerised interviewing has become more popular among survey researchers, primarily because it is believed to improve the quality of survey data and decrease the cost of collection. A large number of empirical studies in the United States and Europe have assessed whether computerised interviews encourage greater reporting of sensitive behaviours. Such studies typically use experimental designs to assign respondents randomly to different interview modes.

Abbreviations: ACASI, audio computer assisted self-interview. 
A wide range of sensitive topics has been examined in these analyses, including sexual behaviour, ${ }^{7-9}$ drug and alcohol use, ${ }^{10-12}$ racial attitudes, ${ }^{13}$ and induced abortion. ${ }^{14}$ Researchers have also investigated the reporting of sexual behaviour and drug use specifically in relation to the risk of sexually transmitted diseases and HIV in both high risk groups ${ }^{15-19}$ and in the general population. ${ }^{20}{ }^{21}$ Although some studies have found little difference between computerised and noncomputerised self-administration, ${ }^{19} 2223$ consistently higher reporting of risky behaviours has usually been observed with computerised methods than with interviewer based methods of data collection.

\section{METHODS}

The data for this paper were obtained from household based surveys in Kisumu district in Nyanza province located some $350 \mathrm{~km}$ northwest of Nairobi. Kisumu was selected for this study because it has one of the highest HIV prevalence rates in Kenya, ${ }^{24}$ heightening the importance of capturing accurate levels of adolescent sexual activity and contraceptive use. The study was based on an experimental design in which never married adolescents were randomly assigned to one of three interview methods: face to face interviews, paper and pencil self-administered interviews, and ACASI interviews. Randomly sampled enumeration areas from which households were selected were drawn using population estimates from the 1999 national census. Fieldwork took place between April and July 2002 and included interviews with approximately 2100 adolescent boys and girls aged 15-21 years; with a sample of this size the power of the significance tests was 0.80 , at an alpha criterion of 0.05 . To achieve the target number of respondents, field workers identified all household members within a selected enumeration area in the days prior to interviewing adolescents. If there was an adolescent in the household, he or she was randomly assigned to one of the three interview modes; only one adolescent was interviewed per household. Respondents were interviewed in private, typically within or just outside their residence. Male and female interviewers were trained for each mode and interviewed only same sex respondents. To facilitate community acceptance, interviewers were recruited from the district, ensuring that they were from the same ethnic group as the respondent and spoke the local language. The interviewers were also relatively young, ranging in age from 20s to mid-30s, and many had previous survey interviewing experience.

The ACASI computer program was configured so that respondents listened to questions and response categories through headphones. Although the questions could have been simultaneously displayed on the computer screen, the computer remained closed during the interview. The respondent answered questions by pressing a number on an external mini keypad that corresponded to a recorded response category.

The questionnaire included 69 questions; two thirds were considered sensitive, asking respondents about their sexual behaviour, alcohol and drug use, contraceptive practice, pregnancies, induced abortions, and births. A face to face exit interview conducted immediately at the end of the main interview queried respondents regarding their feelings about the survey and the interview. The exit interview also included questions for the interviewer to complete concerning the context of the interview-for example, whether anyone else was present during the interview and whether the respondent had trouble completing the questionnaire.

For simplicity of presentation, only the results for the interviewer administered and ACASI surveys are discussed here. Although significant differences in reporting were sometimes found between the self-administered method and the interviewer and ACASI modes in Nyeri and Kisumu, the self-administered method rarely outperformed the other interview modes in obtaining higher levels of reporting of sensitive behaviours. ${ }^{5}$ Thus the self-administered method is of less interest in evaluating the optimal method of eliciting accurate sexual behaviour data. Additionally, in recognition that adolescent girls face greater social pressure to conform to social norms about premarital sex, as well as to explore the issue of response consistency more fully, we restricted ourselves to adolescent girls. Field workers interviewed 349 never married girls aged 15-21 years face to face and 360 using ACASI.

\section{RESULTS}

The questions from the Kisumu survey used for the analyses are listed in table 1. Note that if respondents answered "no" to the question "Have you ever had sexual intercourse?" they were still asked the series of questions about sexual partners and coerced sex. Thus, even when a respondent answered "no" to ever having had sex, she was still asked "Have you ever had sexual intercourse with a boyfriend?"; "Have you ever had sexual intercourse with a stranger?" and so on, with the expectation that she would respond "no" if she had never had sex. Note also that not all the questions are mutually exclusive, particularly the coerced sex questions; for instance, a respondent may have been tricked into having sex by a boyfriend or schoolmate. Additionally, although the sexual partner and coerced sex questions were subsequently asked, they did not immediately follow the "ever had sex" question.

All 349 respondents assigned to the interviewer administered mode provided answers to each sexual behaviour question asked, whereas 53 of the 360 respondents in the ACASI mode refused to answer at least one question. Of the latter, 32 (60\%) had more than one missing value; however, no pattern of missing values was observed. Although the higher response rates in the interviewer administered mode are desirable from an analysis point of view, we believe they are a consequence of the respondents' feeling some pressure to answer the interviewer.

The questions in the survey (see table 1) produce dichotomous indicators of sexual behaviour; hence logistic regression analysis was used to obtain estimates of the differences in reporting by interview mode. Because randomisation may not effectively eliminate all heterogeneity in the sample, the logistic regressions include a set of background covariates in addition to a variable indicating the interview mode. Estimates of the standard errors were adjusted for the clustered nature of the sample. The results

Table 1 Questions from the Kisumu survey used for the analysis

Sexual experience
Have you ever had sexual intercourse?
Sexual partners
Have you ever had sexual intercourse with
a boyfriend?
- a friend, schoolmate, or acquaintance?
a relative?
- a stranger?
Coerced sex 10 or more years older than you?
Have you ever been tricked by a boy into having sex when you did not
want to?
Have you ever been locked in a room by a boy to have sex with you when
you did not want to?
Have you ever been physically forced to have sex when you did not want
to?
Exit interview question
Have you ever had sexual intercourse?


are presented as odds ratios, with the interviewer administered mode serving as the reference category. Predicted percentages were also generated by interview mode from the logistic regression models and are provided for ease of interpretation.

Table 2 presents the odds ratios and predicted probabilities for the estimation of (1) ever had premarital sexual intercourse, (2) sexual intercourse with various types of partners, and (3) coerced sex. Premarital sex is defined by whether respondents, all of whom were never married, reported ever having had sexual intercourse. As can be seen in table 2, for the first two indicators of sexual activityever had sexual intercourse and sexual intercourse with a boyfriend-the difference between interview modes is not significant and the predicted percentages from the models indicate lower reporting with ACASI. The difference in the direction of the predicted values relative to the odds ratio is a function of the distribution of the background covariates by interview mode. For instance, compared with those interviewed face to face, ACASI respondents were significantly more likely to be enrolled in school and to attend church, factors negatively associated with premarital sex. In fact, when the population distributions of these variables are equalised using direct standardisation methods, the predicted percentages of these two behaviours for the ACASI method are slightly higher than the face to face mode, as expected given the odds ratios (results not shown).

The results for the remaining behaviours in table 2 are substantially different from those observed for the ever had sexual intercourse and ever had sexual intercourse with a boyfriend questions. The log odds for each other type of sex partner and coerced sex are significantly greater in the ACASI administration. For instance, ACASI respondents were more than six times as likely to report having had sex with a friend, schoolmate, or acquaintance. Compared with respondents in the interviewer administered group, they were more than three times as likely to report having sex with a stranger or a man 10 or more years older. ACASI respondents were also three times more likely to report coerced sex. The associated predicted percentages from the logistic regressions are equally striking. While it was estimated that only $1 \%$ of young women are predicted to admit to an interviewer that they had sex with a relative, over $20 \%$ are predicted to do so in the computerised interview. If we assume that adolescent girls in Kenya are highly unlikely to exaggerate having had premarital sex, the statistical significance and consistency of these results suggest that ACASI provides a more accurate picture than face to face interviews of the sexual experience of adolescent girls in Kisumu.
Figure 1 illustrates the composition of partners, among those admitting having had sexual intercourse, for each interview mode. The picture that emerges from the interviewer administered surveys is that a majority (57\%) of adolescent girls who have had sexual intercourse, have had sex only with a boyfriend, suggesting limited partnerships. However, this figure drops to $10 \%$ in the ACASI mode. With ACASI, a much wider range of sexual partners is revealed. Also, a much higher incidence of coerced sex is observed, with $45 \%$ of respondents in ACASI reporting having been tricked, locked in a room, or physically forced to have sex. This finding is consistent with levels of sexual coercion found in a national survey of secondary school students aged 12-24 years in Kenya, where $40 \%$ of girls reported that their first sexual encounter was forced. ${ }^{25}$

To explore the issue of response consistency, we compared three different measures of sexual activity in figure 2 . The first pair of bars in the figure shows the predicted percentage of those reporting ever having had sex in answer to the initial general question, as seen in the first column of table 2, while the second pair is constructed from the questions about the specific sex partners. The third measure of sexual activity includes those who reported having had sex with a specific partner, as well as those who reported having been coerced to have sex. The percentage of those having had sex changes very little in the interviewer administered mode, with approximately $48 \%$ of respondents predicted to have had sex across the three measures of sexual activity. However, this pattern is markedly different for ACASI. Although only $43 \%$ of respondents are predicted to report ever having sex, this figure jumps to $61 \%$ when sex with specific partners is used to measure sexual activity and to $68 \%$ when coerced sex is included. These results indicate that the reported levels of premarital sexual activity among adolescent girls in Kisumu are dependent on both the mode of administration and the in-depth probing for various behaviours. The ACASI mode reveals much higher levels of sexual activity when the questions regarding specific sex partners and coerced sex are also considered.

The consistency of responses by method and question type is further explored in table 3, which provides a cross tabulation of the question "Have you ever had sexual intercourse?" by the two alternative measures of sexual activity from figure 2. The diagonals in table 3 represent consistent responses in reporting by interview mode. For instance, the left hand panel considers respondents in the face to face interviews. When a respondent answered "no" to ever had sex and also did not report having had sex with a particular partner, she would fall into the top left hand cell.

Table 2 Odds ratios and predicted percentages from logistic regression of ever had sexual intercourse, sexual partners, and coerced sex*

\begin{tabular}{|c|c|c|c|c|c|c|c|c|c|}
\hline & \multirow{2}{*}{$\begin{array}{l}\text { Ever had } \\
\text { sexual } \\
\text { intercourse }\end{array}$} & \multicolumn{5}{|c|}{ Ever had sexual intercourse with a: } & \multicolumn{3}{|c|}{ Coerced sex } \\
\hline & & Boyfriend & $\begin{array}{l}\text { Friend, schoolmate, } \\
\text { acquaintance }\end{array}$ & Relative & Stranger & $\begin{array}{l}\text { Man } 10 \text { or more } \\
\text { years oldert }\end{array}$ & Tricked† & $\begin{array}{l}\text { Locked in a } \\
\text { room } †\end{array}$ & $\begin{array}{l}\text { Physically } \\
\text { forced } \dagger\end{array}$ \\
\hline \multicolumn{10}{|l|}{ Log odds } \\
\hline Interviewer & 1.00 & 1.00 & 1.00 & 1.00 & 1.00 & 1.00 & 1.00 & 1.00 & 1.00 \\
\hline ACASI & 1.22 & 1.16 & $6.26 \ddagger$ & $42.60 \ddagger$ & $3.45 \ddagger$ & $3.55 \ddagger$ & $3.62 \ddagger$ & $2.74 \ddagger$ & $2.73 \ddagger$ \\
\hline \multicolumn{10}{|l|}{ Predicted (\%) } \\
\hline Interviewer & 48.3 & 45.7 & 9.8 & 1.0 & 3.7 & 3.7 & 7.2 & 5.7 & 6.0 \\
\hline ACASI & 42.8 & 40.1 & 31.9 & 20.9 & 13.9 & 13.9 & 18.9 & 14.0 & 15.8 \\
\hline \multicolumn{10}{|l|}{ Sample sizes } \\
\hline Interviewer & 348 & 348 & 348 & 348 & 348 & 348 & 348 & 348 & 348 \\
\hline ACASI & 302 & 304 & 298 & 302 & 302 & 302 & 297 & 301 & 298 \\
\hline
\end{tabular}

${ }^{*}$ A full set of respondent and household background variables used in estimation of each equation.

†These questions are not mutually exclusive of the sexual partner questions or of each other.

$\neq \mathrm{p}<0.001$; standard errors adjusted for stratification and clustering in the sample design. 

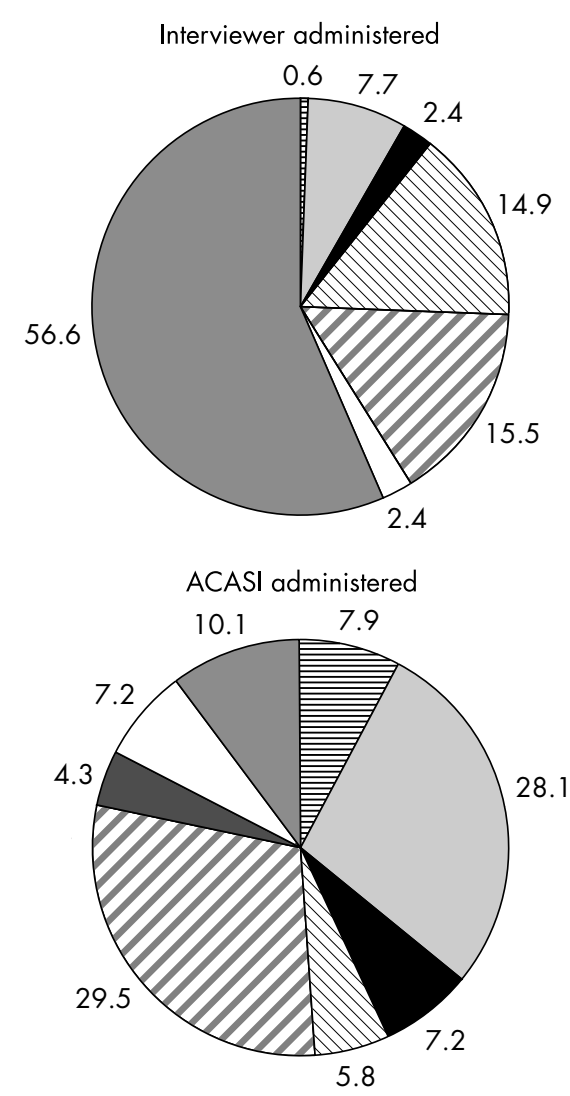

\begin{tabular}{|l}
\hline No partner specified \\
Boyfriend and other and coerced \\
Other and coerced \\
Boyfriend and coerced \\
Boyfriend and other \\
Coerced \\
Other \\
Boyfriend
\end{tabular}

Figure 1 Per cent composition of sexual partners for those reporting ever having sex, by type of interview. "Other" includes ever having had sex with a friend, schoolmate, or acquaintance; relative; stranger; or man 10 or more years older. "Coerced" includes ever have been tricked into sex; locked in a room for sex; or physically forced to have sex.

Conversely, if she responded positively to both the global question and the partner specific questions she would fall into the bottom right hand cell. Alternatively, if a respondent reported that she did not have sex and subsequently admits to having had sex with a particular partner, she would fall into the top of the "off-diagonal", again by interview mode. The bottom off-diagonal represents respondents who reported having had sex, but did not subsequently specify any sexual partner.

In the interviewer administered mode only four respondents are inconsistent across the three measures of sexual activity. For ACASI, a much greater number of cases (94 out of 316) fall into the off-diagonal. The inconsistencies in ACASI are greatest for those who have answered "no" to ever had sex. Of the ACASI respondents who said that they have not had sex, $41 \%$ subsequently indicated that they have had sexual intercourse with a particular partner; this increases to $48 \%$ when coerced sex is considered. Table 3 also reveals a small percentage of inconsistent cases in which ACASI respondents answered affirmatively to the ever had sex question, but then did not indicate a specific partner or that they had been coerced to have sex. It is possible in these cases

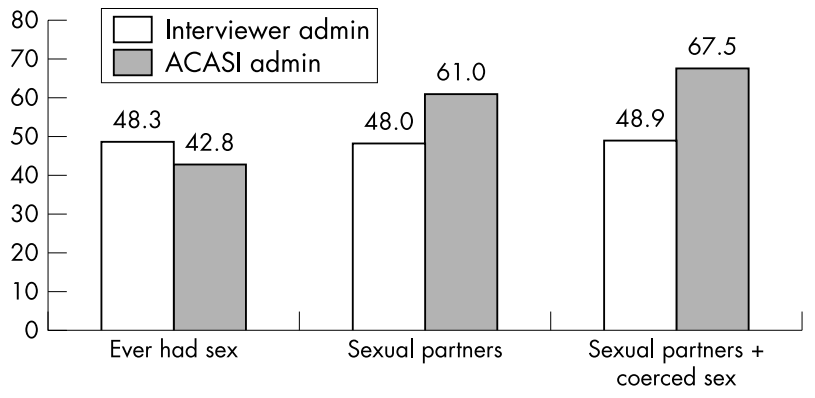

Figure 2 Predicted percentage of those ever having had sex, by measures of sexual activity and type of interview. Note: the sexual partner and coerced sex indicators take on the value of 1 if the respondent answered "yes" to any of the partners listed in table 2, or if she reported having been coerced into sex, even if missing values exist for other sexual partner or coerced sex variables. "Sexual partners" includes ever having had sex with a boyfriend; friend, schoolmate, or acquaintance; relative; stranger; or man 10 or more years older.

"Coerced sex" includes ever having been tricked into sex; locked in a room for sex; or physically forced to have sex.

that the respondents had experienced sexual intercourse with someone other than those listed in the survey.

The degree of consistency in the interviewer administered surveys could be explained by the fact that in denying ever having sexual intercourse, respondents are not likely to subsequently report having sex with a particular partner or being coerced to have sex. This unwillingness to report premarital sexual activity is, of course, the underlying premise for using computerised administration. However, it is also possible that interviewers in the face to face mode may have enforced consistency-either explicitly or tacitly. Having an incentive to finish surveys quickly and to minimise interview dissonance, some interviewers may have asked these questions in a manner that invited a negative response or may have simply filled in the responses themselves. Although specifically trained to ask every question, the exact procedures adopted by interviewers during each interview are largely unknown.

Although the consistency in the interviewer administered mode may be more easily explained, the reasons for the inconsistent responses in ACASI are not. It is clear that given the degree of privacy afforded them in the computerised interview, ACASI respondents found it easier to switch from denying to admitting sexual activity, perhaps knowing that their inconsistent responses were not going to be questioned. But, this begs the question as to why ACASI respondents were not more willing to reveal that they "ever had sexual intercourse" to begin with. Several explanations come to mind, none of which, unfortunately, can be evaluated empirically. One possibility is that respondents became more comfortable over the course of the survey with questions about their sexual behaviour. Although respondents were asked other sensitive questions earlier in the survey, including whether they have ever drunk alcohol, smoked bhang or hashish, and whether their closest unmarried friend had ever had sex, exposure to preliminary questions about their own sexual behaviour may make them more open to probing about sexual partners and coerced sex. A second possibility is that respondents may compartmentalise various sexual behaviours, and therefore not perceive certain sexual activities as being included in the question "Have you ever had sexual intercourse?" Respondents may not feel that being physically forced to have sex or that having sex with a relative or a stranger is the type of sex that is meant when asked whether they have ever had sexual intercourse. In other words, they may perceive the question to mean specifically "Have you ever had sexual intercourse with 
Table 3 Consistency in reporting on sexual behaviour by question type and interview mode

\begin{tabular}{|c|c|c|c|c|c|c|c|c|}
\hline \multirow{3}{*}{$\begin{array}{l}\text { Ever had } \\
\text { sex }\end{array}$} & \multicolumn{4}{|c|}{ Sexual partners* } & \multicolumn{4}{|c|}{ Sexual partners and coerced sex $\dagger$} \\
\hline & \multicolumn{2}{|l|}{ Interviewer } & \multicolumn{2}{|l|}{ ACASI } & \multicolumn{2}{|l|}{ Interviewer } & \multicolumn{2}{|l|}{ ACASI } \\
\hline & No & Yes & No & Yes & No & Yes & No & Yes \\
\hline $\begin{array}{l}\text { No } \\
\text { Yes }\end{array}$ & $\begin{array}{l}181(100) \\
1(0.6)\end{array}$ & $\begin{array}{l}0(0.0) \\
167(99.4)\end{array}$ & $\begin{array}{l}110(59.1) \\
17(11.2)\end{array}$ & $\begin{array}{l}76(40.9) \\
128(88.3)\end{array}$ & $\begin{array}{l}178(98.3) \\
1(0.6)\end{array}$ & $\begin{array}{l}3(1.6) \\
167(99.4)\end{array}$ & $\begin{array}{l}91(52.3) \\
11(7.7)\end{array}$ & $\begin{array}{l}83(47.7) \\
131(92.3)\end{array}$ \\
\hline
\end{tabular}

*Includes questions on ever had sex with boyfriend; friend, schoolmate, or acquaintance; relative; stranger; or man 10 or more years older.

†Coerced sex includes ever been tricked into sex, locked in a room for sex or physically forced to have sex. These variables are not mutually exclusive from the sexual partner questions.

Note: row percentages by interview mode are in parentheses and tally to $100 \%$; consistent responses are shown in italics.

someone you cared for or loved?" This interpretation may explain the highly similar results for this question and for the question "Have you ever had sexual intercourse with a boyfriend?" Although these explanations apply to both modes of administration, it may be that only the computerised interview provides a context in which respondents are comfortable changing their responses.

Not only did we assess consistency within the main interview, we also compared responses in the main interview with those in a face to face exit interview administered immediately after the main survey was completed. Although most questions in the exit interview asked respondents about their perception of the interview (for example, did they feel bored, uncomfortable, or confused? Did they have difficulty completing the questionnaire?), a limited selection of questions from the main survey was asked a second time. Of the sexual behaviour questions, only the question "Have you ever had sexual intercourse?" was repeated in the exit interview, allowing for test-retest comparisons of the consistency of responses by interview mode. For ACASI, it also permits an evaluation of responses in both the computerised and face to face interview settings. If our expectations about the reporting of premarital sex are accurate, we would expect lower levels of reported sexual activity in the face to face exit interview for ACASI respondents.

As can be observed in table 4, contrary to expectations, in the exit interview 55\% of the ACASI respondents reported having had sexual intercourse, a figure $12 \%$ higher than in the main interview and $7 \%$ higher than the proportion reporting having had sex in the interviewer administered mode (see table 2). The higher reporting of sexual behaviour in the ACASI mode is a result of the 54 respondents (or $27 \%$ of those answering "no" in the main interview) who changed their response from "no" to "yes" between the main and exit interviews. These respondents appear on the surface to be

Table 4 Consistency in reporting on sexual behaviour between main and exit interview, by interview mode

\begin{tabular}{|c|c|c|c|c|}
\hline \multirow{3}{*}{$\begin{array}{l}\text { Main survey: ever } \\
\text { had sex }\end{array}$} & \multicolumn{4}{|c|}{ Exit interview: ever had sex } \\
\hline & \multicolumn{2}{|c|}{ Interviewer } & \multicolumn{2}{|l|}{ ACASI } \\
\hline & No & Yes & No & Yes \\
\hline $\begin{array}{l}\text { No } \\
\% \\
\text { Yes } \\
\% \\
\text { Total } \\
\%\end{array}$ & $\begin{array}{l}181 \\
(100) \\
0 \\
0.0 \\
181 \\
(51.9)\end{array}$ & $\begin{array}{l}0 \\
(0.0) \\
168 \\
(100) \\
168 \\
(48.1)\end{array}$ & $\begin{array}{l}143 \\
(72.6) \\
11 \\
(7.5) \\
154 \\
(44.9)\end{array}$ & $\begin{array}{l}54 \\
(27.4) \\
135 \\
(92.5) \\
189 \\
(55.1)\end{array}$ \\
\hline
\end{tabular}

Note: row percentages by interview mode are in parentheses and tally to $100 \%$; consistent responses are shown in italics. more willing to reveal their sexual behaviour to an interviewer. In contrast, 11 respondents $(8 \%$ of those answering "yes" in the main survey) who did not admit to having sex in the face to face exit interview did so in the computerised interview. As suggested above, we had expected this number to be much larger.

It is difficult to understand why some ACASI respondents were willing to disclose in the face to face exit interview that they had had sex but did not do so in the anonymous ACASI context. One possibility is that these respondents in the exit interview were "updating" their responses, given the fact that they had acknowledged having sexual intercourse with at least one sexual partner or having been coerced to having sex (see table 3). Of the 54 respondents in table 4 who responded "yes" to having sex in the exit interview but not in the main survey, $75 \%$ had reported a sexual partner or coerced sex in the computerised interview. However, it is unclear why they were willing to admit this in the face to face interview-perhaps they were concerned that the interviewer was privy to their previous answers. For the remaining $25 \%$ of the 54 respondents, it is hard to pinpoint their motivation for revealing to the interviewer in the exit interview that they had had sex; without further information this inconsistency remains puzzling.

\section{DISCUSSION}

We have argued in this paper and elsewhere ${ }^{5}$ that the interaction between the interviewer and respondent has an effect on answers to sensitive questions. Our review of survey research on adolescent sexual activity in developing countries that has relied on face to face interviews reveals patterns of sexual behaviour that appear inconsistent and implausible. ${ }^{3} 6$ Findings of widely varying levels of sexual activity among boys and girls of similar ages, and of sharply different levels of premarital sex for adolescents across sub-Saharan African countries where one would expect to find similar levels, suggest problems in the reporting of sexual behaviour in adolescent surveys.

Respondents' perceptions of social norms and their own notions of acceptable behaviour influence their willingness to respond candidly to interview questions. In particular, adolescent girls are likely to feel pressure to conform to societal expectations. By modifying their answers to conform to these norms, respondents minimise the gap between their actual behaviour and the perceived social standard of the interviewer. This premise places a premium on the respondents' beliefs regarding such norms and their perceptions of the interviewer's opinions and values. ${ }^{27}$ Using various social cues, such as gender, age, ethnic group, and education, and inferences derived from verbal and non-verbal cues of the interviewer, the respondents are likely to adjust their answers to minimise any dissonance generated in the context of the interview. 
The results outlined in this paper present fresh evidence that both the mode of survey administration and the probing for various behaviours significantly affect the observed prevalence of sexual activity. However, as was indicated, these effects are not always predictable or easy to explain. For instance, the fact that ACASI does not perform significantly better than the interviewer administered mode for the initial "ever had sexual intercourse" and "ever had sexual intercourse with a boyfriend" questions, suggests that computerised administration is not a panacea to underreporting of sexual behaviour.

For the highly stigmatising sexual behaviour questions, the results were clearer. Respondents were significantly more likely to report in ACASI that they had had sex with a relative, stranger, or older man, and/or had been coerced into having sex. Given our assumption that girls do not overreport sexual behaviour of a highly stigmatising nature, higher levels of reporting imply greater accuracy in reporting. ACASI produced a more diverse picture of adolescent sexual activity than the face to face interviewer administered method, a picture which suggests that adolescent girls in Kenya have more complicated, and clearly more perilous sex lives than traditional surveys of sexual activity indicate. Our findings reinforce evidence found elsewhere that sexual coercion is a significant component of the adolescent sexual experience in developing countries. ${ }^{28}$ If accurate, these findings have implications for programme managers and policy makers seeking to meet the reproductive health needs of young women in sub-Saharan Africa.

\section{ACKNOWLEDGEMENTS}

The authors gratefully acknowledge the role of the project supervisors, Francis Ayuka (in Kisumu) and Arjmandbanu Khan (in Nairobi), as well as the 18 interviewers and three supervisors who carried out the field research in Kisumu. They also recognise the technical assistance provided by Stanley Mierzwa at the Population Council, New York, and Mike Shamku at the Population Council, Nairobi.

Funding for this research was provided by the National Institute of Child Health and Human Development (Grant R01 HD35700-02); by the Office of Population, Bureau for Global Health, US Agency for International Development, under the terms of Award No. HRN-A00-99-00010; and by the Bill and Melinda Gates Foundation.

\section{CONTRIBUTORS}

PCH and BSM wrote the manuscript with comments, suggestions, and contributions by ASE. PCH carried out the empirical analyses and assisted in the monitoring and oversight of the data collection. ASE supervised and implemented the data collection.

\section{Authors' affiliations \\ P C Hewett, B S Mensch, Population Council, New York, USA \\ A S Erulkar, Population Council, Ghana}

\section{REFERENCES}

1 UNAIDS. UNAIDS fact sheet on differences in HIV spread in African cities: AIDS in Africa, 1998: Available at http://www.unaids.org/publications/ documents/epidemiology/determinants/saepap98.html (accessed 16 June 2000).

2 UNAIDS, WHO. Epidemiological fact sheet on HIV/AIDS and sexually transmitted diseases: Kenya, 1998: Available at http://www.who.int/emchiv/fact_sheets.html (accessed 16 June 2000).
3 Zaba B, Boerma JT, Pisani E, et al. Estimation of levels and trends in age at first sex from African demographic surveys using survival analysis. Paper presented at the annual meeting of the Population Association of America, Atlanta, 9-11 May, 2002.

4 Glynn JR, Carael $M$, Auvert B, et al. Why do young women have a much higher prevalence of HIV than young men? A study in Kisumu, Kenya and Ndola, Zambia. AIDS 2001;15(Suppl 4):S51-S60.

5 Mensch BS, Hewett PC, Erulkar AS. The reporting of sensitive behaviour by adolescents: A methodological experiment in Kenya. Demography 2003;40:247-68

6 Couper MP, Nicholls II WL. The history and development of computer-assisted survey information collection methods. In: Couper MP, Baker RP, Bethlehem J, et al. Computer assisted information collection. New York: John Wiley, 1998: 1-22.

7 Turner CF, Ku L, Rogers M, et al. Adolescent sexual behaviour, drug use and violence: increased reporting with computer survey technology. Science 1998;280:867-73.

8 Tourangeau R, Smith TW. Asking sensitive questions: the impact of data collection mode, question format, and question context. Public Opin $Q$ 1996;60:275-304.

9 Rumakom P, Guest P, Chinvarasopak W, et al. Obtaining accurate responses to sensitive questions: a comparison of two data collection techniques. Draft. Bangkok: Population Council, 1999.

10 Aquilino WS. Interview mode effects in surveys of drug and alcohol use: a field experiment. Public Opin Q 1994;58:210-40.

11 Aquilino WS, LoScuito LA. Effects of interviewer mode on self-reported drug use. Public Opin Q 1990;54:362-95.

12 Beebe TJ, Harrison PA, Mcrae JA, et al. An evaluation of computer-assisted self-interviews in a school setting. Public Opin $Q$ 1998;62:623-32.

13 Kyrsan M. Privacy and the expression of white racial attitudes: a comparison across three contexts. Public Opin Q 1998;62:506-44.

14 Fu H, Darroch JE, Henshaw S, et al. Measuring the extent of abortion underreporting in the 1995 National Survey of Family Growth. Fam Plann Perspect 1998;30:128-38.

15 Macalino G, Celentano D, Latkin C, et al. Risk behaviours by audio computerassisted self-interviews among HIV-seropositive and HIV-seronegative injection drug users. AIDS Educ Prev 2002; 14:367.

16 Metzger DS, Koblin B, Turner CF, et al. Randomized controlled trial of audio computer-assisted self-interviewing: utility and acceptability in longitudinal studies. HIVNET Vaccine Preparedness Study Protocol Team. Am J Epidemiol 2000;152:99-106.

17 Williams ML, Freeman RC, Brown AM, et al. A comparison of the reliability of self-reported drug use and sexual behaviours using computer-assisted versus face-to-face interviewing. AIDS Educ Prev 2000;12:199-213.

18 Des Jarlais DC, Paone D, Miliken J, et al. Audio-computer interviewing to measure risk behaviour for HIV among injecting drug users: a quasirandomised trial. Lancet 1999;353:1657-61.

19 Boekeloo BO, Schiavo L, Rabin DL, et al. Self-reports of HIV risk factors by patients at a sexually transmitted disease clinic: audio vs. written questionnaires. Am J Public Health 1994:8:754-60.

20 Johnson AM, Copas AJ, Erens Bm, et al. Effect of computer-assisted self-interviews on reporting of sexual HIV risk behaviours in a general population sample: a methodological experiment. AIDS 2001;15:111-15.

21 Copas AJ, Johnson AM, Wadsworth J. Assessing participation bias in a sexual behaviour survey: implications for measuring HIV risk. AIDS 1997; 11:783-90

22 Jobe JB, Pratt WF, Tourangeau R, et al. Effects of interview mode on sensitive questions in a fertility survey. Lyberg L. Survey measurement and process quality. New York: Wiley, 1997:311-29.

23 Millstein SG, Irwin CE. Acceptability of computer-acquired sexual histories in adolescent girls. J Pediatr 1983;103:815-19.

24 Buvé A, Caraël M, Hayes RJ, et al. Multicentre study on factors determining differences in rate of spread of HIV in sub-Saharan Africa: methods and prevalence of HIV infection. AIDS 2001;15(Suppl 4):S5-S14.

25 In: Youri P, ed. Female adolescent health and sexuality in Kenyan secondary schools: a research report. Unpublished report. African Medical and Research Foundation, Nairobi, 1994.

26 Mensch BS, Clark WH, Lloyd CB, et al. Premarital sex, schoolgirl pregnancy and school quality in rural Kenya. Stud Fam Plann 2001;32:285-301.

27 Sudman S, Bradburn NM, Schwarz N. Modest expectations: the effects of interviewers' prior expectations on responses. Sociol Method Res 1977:6:171-82.

28 Jejeebhoy SJ, Bott S. Non-consensual sexual experiences of young people: a review of the evidence from developing countries. Population Council Regional Working Paper: South and East Asia, 16. New Delhi, 2003. 